Stud. Univ. Babeş-Bolyai Math. 64(2019), No. 3, 331-338

DOI: http://dx.doi.org/10.24193/subbmath.2019.3.04

\title{
Iterates of positive linear operators on Bauer simplices
}

\author{
Mădălina Dancs and Sever Hodiş
}

Dedicated to Professor Heiner Gonska on the occasion of his 70th anniversary.

\begin{abstract}
We consider positive linear operators acting on $C(K)$, where $K$ is a metrizable Bauer simplex. For such an operator $L$ we investigate the limit of the iterates $L^{m}$, when $m \rightarrow \infty$. Qualitative results and rates of convergence are obtained. The general results are illustrated by examples involving classical operators.
\end{abstract}

Mathematics Subject Classification (2010): 41A36, 46A55.

Keywords: Bauer simplex, positive linear operators, iterates, convergence.

\section{Introduction}

Iterates of positive linear operators were investigated in many papers and from several points of view. General criteria for their convergence can be found in [1], [2], [13], [14], [20], [21], [23]. Rates of convergence of the iterates were established in $[6],[10],[16],[17],[18],[20],[21],[28]$. The relationship with Korovkin theory is presented in [6], [7], [8], [22]. Iterates are essentially used for representing some strongly continuous semigroups of operators: see [7], [8], [17], [28]. Iterates for qBernstein operators are studied in [24]; the case of complex operators is considered in [11]. In the above papers analytical methods were used and also methods from probability theory. Results based on spectral theory can be found in [9]; fixed point theory is used in [3], [4], [30], [31], [32], [33].

This paper is devoted to the study of iterates of positive linear operators on Bauer simplices. General definitions and results are presented in this introduction; see also [5], [7], [8], [25].

Section 2 is devoted to the iterates of operators preserving affine functions. An example concerning a finite dimensional simplex is discussed in Section 3. Other examples are presented in Section 4.

Throughout the paper we shall use the following notions. 
Let $E$ be a real locally convex Hausdorff space and $K$ a convex compact subset of $E$. Let $C(K)$ be the space of all continuous real-valued functions on $K$, endowed with the usual ordering and the supremum norm. By Hervé's theorem [5, Th.I.4.3], [7, p.57], $C(K)$ contains a strictly convex function if and only if $K$ is metrizable. Throughout the paper we shall suppose that $K$ is metrizable.

The set of all probability Radon measures on $K$ will be denoted by $M_{1}^{+}(K)$. For each $x \in K, \varepsilon_{x}$ stands for the probability Radon measure concentrated on $\{x\}$.

The Choquet-Meyer ordering $<$ on $M_{1}^{+}(K)$ is defined as follows: for every $\mu, \nu \in$ $M_{1}^{+}(K), \mu<\nu$ if $\mu(f) \leq \nu(f)$ for every convex function $f \in C(K)$. A measure $\mu$ which is maximal with respect to $<$ will be simply called a maximal measure.

Let $A(K)$ be the set of all affine functions for all $h \in C(K)$. The barycenter of $\mu \in M_{1}^{+}(K)$ is the point $r \in K$ for which $\mu(h)=h(r), h \in A(K)$; in this case $\mu(f) \geq f(r)$ for each convex function $f \in C(K)$.

There are several equivalent properties defining a Choquet simplex. We need the following one:

$K$ is called a Choquet simplex if for every $x \in K$ there exists a unique maximal measure $\mu_{x} \in M_{1}^{+}(K)$ having $x$ as barycenter.

The set of the extreme points of $K$ will be denoted by $e x(K)$.

A Choquet simplex $K$ such that $\operatorname{ex}(K)$ is closed will be called a Bauer simplex. In this case $\mu_{x}$ is supported by $\operatorname{ex}(K)$; moreover, if $\mu_{x}=\varepsilon_{x}$, then $x \in \operatorname{ex}(K)$.

If $K$ is a Bauer simplex, then the operator $P: C(K) \longrightarrow A(K)$ defined by

$$
P f(x)=\mu_{x}(f), f \in C(K), x \in K,
$$

is linear, positive, and $P h=h$ for all $h \in A(K)$.

$P$ is called the canonical projection associated with the Bauer simplex $K$.

Let $L: C(K) \longrightarrow C(K)$ be a positive linear operator such that $L h=h$, for every $h \in A(K)$. For each $x \in K$ let $\nu_{x}(f):=L f(x), f \in C(K)$. Then $\nu_{x} \in M_{+}^{1}(K)$ and $x$ is the barycenter of $\nu_{x}$. In particular, if $x \in e x(K)$ then $\nu_{x}=\varepsilon_{x}$, so that

$$
L f(x)=f(x), x \in e x(K), f \in C(K) .
$$

Moreover, if $g \in C(K)$ is convex, then $\nu_{x}(g) \geq g(x), x \in K$, i.e.,

$$
L g \geq g \text {. }
$$

We shall need the following result [26], [27], [7, Th.1.5.2].

Lemma 1.1. Let $\mu \in M_{1}^{+}(K)$ with barycenter $r$ and let $u$ be a strictly convex function. If $\mu(u)=u(r)$, then $\mu=\varepsilon_{r}$.

\section{Iterates of positive linear operators preserving the affine functions}

In the sequel, $K$ will be a metrizable Bauer simplex.

Theorem 2.1. Let $L: C(K) \longrightarrow C(K)$ be a positive linear operator such that $L h=h$, $h \in A(K)$. Let $u \in C(K)$ be a strictly convex function. If

$$
\lim _{m \rightarrow \infty} L^{m} f=P f, f \in C(K),
$$


then

$$
L u(x)>u(x), x \in K \backslash e x(K) .
$$

Proof. Let $x \in K$. As in the preceding section, let $\nu_{x}(f):=L f(x), f \in C(K)$. By $(1.2), L u(x) \geq u(x)$. Suppose that $L u(x)=u(x)$. Then $\nu_{x}(u)=u(x)$, and Lemma 1.1 yields $\nu_{x}=\varepsilon_{x}$, i.e., $L f(x)=f(x), f \in C(K)$. By induction, $L^{m} f(x)=f(x)$, $f \in C(K)$. Now (2.1) shows that $P f(x)=f(x), f \in C(K)$. This means that $\mu_{x}=\varepsilon_{x}$, which entails $x \in \operatorname{ex}(K)$.

For $K=[0,1]$ the above result was obtained in [29] and [12, Corollary 2].

We shall prove that the converse of Th. 2.1 is also true. Having applications in mind, let us consider a sequence of positive linear operators $L_{n}: C(K) \longrightarrow C(K)$ preserving the affine functions, i.e.,

$$
L_{n} h=h, h \in A(K), n \geq 1 .
$$

Fix a strictly convex function $u \in C(K)$.

For $n \geq 1$ and $s \in(0,+\infty)$ define

$$
a_{n}(s):=\max _{K}\left(P u-u-n s\left(L_{n} u-u\right)\right) .
$$

For $x \in e x(K)$ we have $P u(x)-u(x)=L_{n} u(x)-u(x)=0$, so that $a_{n}(s) \geq 0$.

Lemma 2.2. If $n s \geq 1, m \geq 1$, then

$$
0 \leq P u-L_{n}^{m} u \leq a_{n}(s) \mathbf{1}+\left(1-\frac{1}{n s}\right)^{m}(P u-u),
$$

where 1 is the constant function of value 1 defined on $K$.

Proof. Since $P$ preserves the affine functions, we have $u \leq P u$ by virtue of (1.2). Moreover, $P u \in A(K)$, and so $L_{n} u \leq L_{n}(P u)=P u$. By induction we get $L_{n}^{m} u \leq P u$, and this is the first inequality in (2.5).

From (2.4) we derive

$$
a_{n}(s) \mathbf{1} \geq P u-u-n s\left(L_{n} u-u\right)
$$

This implies

$$
\frac{1}{n s}\left(P u-a_{n}(s) \mathbf{1}\right)+\left(1-\frac{1}{n s}\right) u \leq L_{n} u .
$$

Since $1-\frac{1}{n s} \geq 0$, iterating over $m \geq 1$

$$
\left(1-\left(1-\frac{1}{n s}\right)^{m}\right)\left(P u-a_{n}(s) \mathbf{1}\right)+\left(1-\frac{1}{n s}\right)^{m} u \leq L_{n}^{m} u .
$$

This leads immediately to the second inequality in (2.5), and the lemma is proved.

Lemma 2.3. Let $n \geq 1$ be fixed, and suppose that for a given strictly convex function $u \in C(K)$ one has

$$
L_{n} u(x)>u(x), x \in K \backslash e x(K) .
$$

Then $\lim _{s \rightarrow \infty} a_{n}(s)=0$. 
Proof. Since $a_{n} \geq 0$ and $a_{n}$ is decreasing on $(0,+\infty)$, we have $l:=\lim _{s \rightarrow \infty} a_{n}(s) \geq 0$. Suppose that $l>0$. Let

$$
A_{s}:=\left\{x \in K: P u(x)-u(x)-n s\left(L_{n} u(x)-u(x)\right) \geq l\right\} .
$$

The sets $A_{s}$ are closed and the family $\left(A_{s}\right)_{s>0}$ is descending. For each $s>0, A_{s}$ and ex $(K)$ are disjoint, so that (2.6) implies $\bigcap_{s>0} A_{s}=\emptyset$. Since $K$ is compact, there exists $t>0$ such that $A_{t}=\emptyset$. Then $a_{n}(t)<l$, a contradiction.

Theorem 2.4. (i) Let $0<c<1$. Then

$$
0 \leq P u-L_{n}^{m} u \leq a_{n}\left(m^{c}\right) \mathbf{1}+\left(1-\frac{1}{n m^{c}}\right)^{m}(P u-u),
$$

for all $m, n \geq 1$.

(ii) If (2.6) holds for a given $n \geq 1$, then

$$
\lim _{m \rightarrow \infty} L_{n}^{m} f=P f, f \in C(K) .
$$

Proof. (i) is a consequence of (2.5), with $s=m^{c}$. From (2.7) and Lemma 2.2 we infer that $\lim _{m \rightarrow \infty} L_{n}^{m} u=P u$. This fact, combined with Corollary 3.3 .4 of [7], leads to (2.8).

In the sequel we shall suppose that the limit

$$
T(t) f:=\lim _{n \rightarrow \infty} L_{n}^{k(n)} f
$$

exists in $C(K)$ for each $f \in C(K)$, each $t \geq 0$, and each sequence of positive integers $(k(n))_{n \geq 1}$ such that $\lim _{n \rightarrow \infty} \frac{k(n)}{n}=t$.

Denote $a(s)=\sup \left\{a_{n}(s): n \geq 1\right\}, s>0$.

Theorem 2.5. (i) Let $0<c<1$. Then for all $t>0$,

$$
0 \leq P u-T(t) u \leq a\left(t^{c}\right) \mathbf{1}+(P u-u) \exp \left(-t^{1-c}\right) .
$$

(ii) If $\lim _{s \rightarrow \infty} a(s)=0$, then

$$
\lim _{t \rightarrow \infty} T(t) f=P f, f \in C(K) .
$$

Proof. Let $t>0$ be fixed. If $n t^{c} \geq 1$, from (2.5) we get

$$
0 \leq P u-L_{n}^{k(n)} u \leq a\left(t^{c}\right) \mathbf{1}+\left(1-\frac{1}{n t^{c}}\right)^{k(n)}(P u-u) \text {. }
$$

Choosing $k(n)$ such that $\lim _{n \rightarrow \infty} \frac{k(n)}{n}=t$, we obtain $(2.9)$.

If $\lim _{s \rightarrow \infty} a(s)=0,(2.9)$ yields

$$
\lim _{t \rightarrow \infty} T(t) u=P u \text {. }
$$

Another application of [7, Cor. 3.3.4] concludes the proof. 


\section{An example and a quantitative result}

Let $K$ be the canonical simplex of $\mathbb{R}^{d}$, that is

$$
K=\left\{x \in \mathbb{R}^{d}: x_{1}, \ldots x_{d} \geq 0, x_{1}+\ldots+x_{d} \leq 1\right\} .
$$

The canonical projection associated with $K$ is defined, for every $f \in C(K)$ and $x \in K$, by

$$
P f(x)=\left(1-x_{1}-\ldots-x_{d}\right) f(0)+x_{1} f\left(v_{1}\right)+\ldots+x_{d} f\left(v_{d}\right),
$$

where $0, v_{1}:=(1,0, \ldots, 0), \ldots, v_{d}:=(0, \ldots, 0,1)$ are the vertices of $K$.

Let $f \in C(K)$; suppose that there exists a constant $Q_{f}>0$ such that for all $x \in K$,

$$
\begin{gathered}
|f(x)-f(0)| \leq Q_{f} \sum_{i=1}^{d} x_{i} \\
\left|f(x)-f\left(v_{j}\right)\right| \leq Q_{f}\left(1-2 x_{j}+\sum_{i=1}^{d} x_{i}\right), j=1, \ldots, d .
\end{gathered}
$$

Then, for $x \in K$,

$$
\begin{gathered}
|f(x)-P f(x)|=\left|f(x)-\left(1-\sum_{i=1}^{d} x_{i}\right) f(0)-\sum_{i=1}^{d} x_{i} f\left(v_{i}\right)\right| \\
=\left|\left(1-\sum_{i=1}^{d} x_{i}\right)(f(x)-f(0))+\sum_{i=1}^{d} x_{i}\left(f(x)-f\left(v_{i}\right)\right)\right| \\
\leq Q_{f}\left(\left(1-\sum_{i=1}^{d} x_{i}\right) \sum_{i=1}^{d} x_{i}+\sum_{i=1}^{d} x_{i}\left(1-2 x_{i}+\sum_{j=1}^{d} x_{j}\right)\right) \\
=2 Q_{f}\left(\sum_{i=1}^{d} x_{i}-\sum_{i=1}^{d} x_{i}^{2}\right) .
\end{gathered}
$$

Consider the strictly convex function $u \in C(K), u(x)=\sum_{i=1}^{d} x_{i}^{2}, x \in K$. Then

$$
P u(x)=\sum_{i=1}^{d} x_{i}
$$

so that for the above function $f$ we have

$$
|f(x)-P f(x)| \leq 2 Q_{f}(P u(x)-u(x)), x \in K .
$$

Let $L_{n}: C(K) \longrightarrow C(K)$ be a positive linear operator preserving affine functions. From (3.4) we get

$$
\left|L_{n}^{m} f-P f\right| \leq 2 Q_{f}\left(P u-L_{n}^{m} u\right) .
$$

Finally, (3.5) and (2.7) yield

$$
\left|L_{n}^{m} f-P f\right| \leq 2 Q_{f}\left(a_{n}\left(m^{c}\right) \mathbf{1}+\left(1-\frac{1}{n m^{c}}\right)^{m}(P u-u)\right),
$$


i.e.,

$$
\left|L_{n}^{m} f(x)-P f(x)\right| \leq 2 Q_{f}\left[a_{n}\left(m^{c}\right)+\left(1-\frac{1}{n m^{c}}\right)^{m} \sum_{i=1}^{d} x_{i}\left(1-x_{i}\right)\right] .
$$

Moreover, in the context of Theorem 2.3 we derive from (3.4):

$$
|T(t) f-P f| \leq 2 Q_{f}(P u-T(t) u) .
$$

Combined with (2.9), this gives

$$
|T(t) f(x)-P f(x)| \leq 2 Q_{f}\left[a\left(t^{c}\right)+\left(\exp \left(-t^{1-c}\right)\right) \sum_{i=1}^{d} x_{i}\left(1-x_{i}\right)\right] .
$$

Remark 3.1. If $f \in C^{1}(K)$, i.e., $f$ has continuous partial derivatives on the interior of $K$ which can be continuously extended on $K$, then (3.2) and (3.3) are satisfied with

$$
Q_{f}:=\max \left\{\left\|\frac{\partial f}{\partial x_{i}}\right\|_{\infty}: i=1, \ldots, d\right\} .
$$

\section{Examples}

In this section we present examples of sequences $\left(L_{n}\right)_{n \geq 1}$ of operators preserving affine functions and satisfying the fundamental condition (2.6).

Example 4.1. Let $B_{n}, n \geq 1$, be the Bernstein-Schnabl operators associated with the canonical projection $P$ and the arithmetic mean Toeplitz matrix (see [7, p. 381]). Let $u \in C(K)$ be a strictly convex function. Suppose that for a given $n \geq 1$ and a given $x \in K$ one has $B_{n} u(x)=u(x)$. According to Lemma 1.1, we infer that $B_{n} f(x)=f(x)$, for every $f \in C(K)$. In particular, $B_{n} h^{2}(x)=h^{2}(x)$, for all $h \in A(K)$. Now [7, (6.1.16)] leads to $P\left(h^{2}\right)(x)=h^{2}(x), h \in A(K)$. From [7, Cor. 3.3.4 and Remark to Prop. 3.3.2] we deduce that $x \in e x(K)$. So (2.6) is satisfied for the operators $B_{n}$.

Example 4.2. Let $U_{n}, n \geq 1$, be the genuine Bernstein-Durrmeyer operators on a simples $K$ in $\mathbb{R}^{d}$ (see [34], [19], [35]). If $u \in C(K)$ is strictly convex, then $U_{n} u \geq$ $B_{n} u \geq u\left[19\right.$, Th.8]. If $U_{n} u(x)=u(x)$, then $B_{n} u(x)=u(x)$, and from Ex. 4.1 we know that $x \in \operatorname{ex}(K)$. So $(2.6)$ is satisfied for the operators $U_{n}$.

Example 4.3. It was proved in [28, Example 2.4] that (2.6) is satisfied for the classical Meyer-König and Zeller operators on $C[0,1]$.

Example 4.4. The case of the Bernstein-Schnabl operators on the unit interval, associated with a continuous selection of probability Borel measures on $[0,1]$, is considered in [28, Example 3.3]. The operators satisfy (2.6).

For all the operators presented in the above examples one can apply Lemma 2.2 and, consequently, one can obtain the corresponding quantitative results derived from Theorems 2.2 and 2.3 .

Other examples and quantitative results can be found in [18], [28], [29]. 


\section{References}

[1] Agratini, O., On the iterates of a class of summation-type linear positive operators, Comput. Math. Appl., 55(2008), 1178-1180.

[2] Agratini, O., On some Bernstein type operators: iterates and generalizations, East J. Approx., 9(2003), 415-426.

[3] Agratini, O., Rus, I.A., Iterates of a class of discrete linear operators via contraction principle, Comment. Math. Univ. Carolin., 44(2003), no. 3, 555-563.

[4] Agratini, O., Rus, I.A., Iterates of some bivariate approximation process via weakly Picard operators, Nonlinear Anal. Forum, 8(2003), 159-168.

[5] Alfsen, E., Compact Convex Sets and Boundary Integrals, Springer 1971.

[6] Altomare, F., On some convergence criteria for nets of positive operators on continuous function spaces, J. Math. Anal. Appl., 398(2013), 542-552.

[7] Altomare, F., Campiti, M., Korovkin-Type Approximation Theory and its Applications, Walter de Gruyter, Berlin, New York, 1994.

[8] Altomare, F., Cappelletti Montano, M., Leonessa, V., Raşa, I., Markov Operators, Positive Semigroups and Approximation Processes, De Gruyter Studies in Mathematics, 61, 2014.

[9] Badea, C., Bernstein polynomials and operator theory, Results Math., 53(2009), 229-236.

[10] Birou, M., New rates of convergence for the iterates of some positive linear operators, Mediterr. J. Math., (2017), 14-129.

[11] Gal, S.G., Voronovskaja's theorem and iterations for complex Bernstein polynomials in compact disks, Mediterr. J. Math., 5(3)(2008), 253-272.

[12] Gavrea, I., Ivan, M., On the iterates of positive linear operators, J. Approx. Theory, 163(2011), 1076-1079.

[13] Gavrea, I., Ivan, M., On the iterates of positive linear operators preserving the affine functions, J. Math. Anal. Appl., 372(2010), 366-368.

[14] Gavrea, I., Ivan, M., The iterates of positive linear operators preserving the constants, Appl. Math. Lett., 24(12)(2011), 2068-2071.

[15] Gavrea, I., Ivan, M., Asymptotic behaviour of the iterates of positive linear operators, Abstr. Appl. Anal., (2011), art. ID 670509.

[16] Gonska, H., Kacsó, D., Piţul, P., The degree of convergence of over-iterated positive linear operators, J. Appl. Funct. Anal., 1(2006), 403-423.

[17] Gonska, H., Raşa, I., The limiting semigroup of the Bernstein iterates: degree of convergence, Acta Math. Hungar., 111(2006), 119-130.

[18] Gonska, H., Raşa, I., On infinite products of positive linear operators reproducing linear functions, Positivity, 17(2013), no. 1, 67-79.

[19] Goodman, T.N.T., Sharma, A., A Bernstein type operator on the simplex, Math. Balkanica, 5(1991), 129-145.

[20] Mahmudov, N.I., Asymptotic properties of iterates of certain positive linear operators, Math. Comput. Model., 57(2013), 1480-1488.

[21] Mahmudov, N.I., Asymptotic properties of powers of linear positive operators which preserve $e_{2}$, Comput. Math. Appl., 62(2011), 4568-4575.

[22] Mahmudov, N.I., Korovkin type theorem for iterates of certain positive linear operators, arXiv:1103.2918v1. 
[23] Micchelli, C.A., The saturation class and iterates of the Bernstein polynomials, J. Approx. Theory, 8(1973), 1-18.

[24] Ostrovska, S., q-Bernstein polynomials and their iterates, J. Approx. Theory, 123(2003), 232-255.

[25] Phelps, R.R., Lectures on Choquet's Theorem, Springer, 2001.

[26] Raşa, I., Sets on which concave functions are affine and Korovkin closures, Anal. Numér. Théor. Approx., 15(1986), no. 2, 163-165.

[27] Raşa, I., On some results of C. A. Micchelli, Anal. Numér. Théor. Approx., 9(1980), no. $1,125-127$.

[28] Raşa, I., $C_{0}$-semigroups and iterates of positive linear operators: asymptotic behaviour, Rend. Circ. Mat. Palermo (2) Suppl., 82(2010), 123-142.

[29] Raşa, I., Asymptotic behaviour of iterates of positive linear operators, Jaen J. Approx., 1(2009), no. 2, 195-204.

[30] Rus, I.A., Picard operators and applications, Sci. Math. Japon., 58(2003), no. 1, 191-219.

[31] Rus, I.A., Iterates of Stancu operators, via contraction principle, Stud. Univ. BabeşBolyai Math., 47(2002), no. 4, 101-104.

[32] Rus, I.A., Iterates of Stancu operators (via fixed point principles) revisited, Fixed Point Theory, 11(2010), no. 2, 369-374.

[33] Rus, I.A., Iterates of Bernstein operators, via contraction principle, J. Math. Anal. Appl., 292(2004), 259-261.

[34] Sauer, T., The Genuine Bernstein-Durrmeyer operator on a simplex, Results Math., 26(1994), no. 1-2, 99-130.

[35] Waldron, Sh., A generalised beta integral and the limit of the Bernstein-Durrmeyer operator with Jacobi weights, J. Approx. Theory, 122(2003), 141-150.

Mădălina Dancs

Technical University of Cluj-Napoca

Department of Mathematics

28, Memorandumului Street

400114 Cluj-Napoca, Romania

e-mail: dancs_madalina@yahoo.com

Sever Hodiş

Technical University of Cluj-Napoca

Department of Mathematics

28, Memorandumului Street

400114 Cluj-Napoca, Romania

e-mail: hodissever@gmail.com 